\title{
Comparison of Breastfeeding Knowledge Before and After Studying Obstetric Course at Nursing College
}

\author{
Zeinab Ahmed Abu Sabeib* and Samira Ahmed Alsenany \\ Princess Nourah Bint Abdulrahman University/College of Nursing, Riyadh, \\ Saudi Arabia
}

\begin{abstract}
Article Type: Article
Article Citation: Zeinab Ahmed Abu Sabeib, Samira Ahmed Alsenany. Comparison of breastfeeding knowledge before and after studying obstetric course at nursing college. Indian Journal of Science and Technology. 2020; 13(04), 400-404. D0l: 10.17485/ijst/2020/ v013i04/149141
\end{abstract}

Received date: March 13, 2019

Accepted date: April 2, 2019

*Author for correspondence: Zeinab Ahmed Abu Sabeib $\mathbf{\nabla}$

zaabusabeib@pnu.edu.sa Princess Nourah Bint Abdulrahman University/ College of Nursing, Riyadh, Saudi Arabia

\begin{abstract}
Background: Breastfeeding is a significant health issue; it is natural, safe, and nutritive for the babies. Objectives: The study aim was to assess nursing college students' knowledge and attitudes about breastfeeding before and after studying an obstetrics course at a university in Riyadh, Kingdom of Saudi Arabia. Methods: An exploratory descriptive study done in March 2017, in the College of Nursing. A convenient sample (94 and 63 students from levels 5 and 7, respectively) included in the study. A self-administered questionnaire was used to assess the students' knowledge and attitudes, and to compare the results of those who studied the obstetric course and those who have not. Results: No significant difference was found between the two levels regarding the score of knowledge and attitude. Conclusion: Realization of the significance of breastfeeding should be promoted among nursing students through comprehensive training.
\end{abstract}

Keywords: Breastfeeding, Nursing, Obstetric Course, Students.

\section{Introduction}

It is known worldwide that breastfeeding is a significant health issue; it is natural, safe, and nutritive for the babies. Scariati and others stated that if babies are exclusively breastfed for four months they are protected from diarrhea as breast milk has positive effects on intestinal bacteria and also helps in the protection from ear infections [1]. Walker mentioned that the feeding formula is linked to different conditions such as immune system disorders, respiratory, and ear infections. In addition, Bachrach and others reported that breastfed infants suffer from fewer severe respiratory tract diseases compared to those who are not [2]. Breastfeeding also reinforces the underdeveloped immune system and supports the host defense mechanisms against infections [3]. Horwood and Fergusson concluded that breastfeeding promotes infants' cognitive skills and intelligence quotients when they grow older [4]. Moreover, Weiner agreed that breastfeeding is more economical for the 
families and the government as breastfed infants catch less common infections, so that the expenses of the health care will be lesser for the breastfed babies [5].

World Health Organization and the American Dietetic Association encourage exclusive breastfeeding in the first six months and continued breastfeeding with complimentary food up to 1 year [6]. Even with the great efforts to foster breastfeeding, the average in Saudi Arabia has stayed low [7]. It has been stated that in Saudi Arabia, at the age of 3 months exclusive breastfeeding is dropping from $90 \%$ to 50\%, and it was reported in 1998 that; breastfeeding continuance up to 2 years was declined in 2008 from $32 \%$ to $3.2 \%$ [8].

Many factors contribute to that declining tendency, including the help of the breastfeeding mothers in the early weeks of breastfeeding by providers of healthcare, and the providing of adequate information to the mothers [9]. The lack of assistance by healthcare providers and inadequate and incorrect information can affect the mothers' attitudes towards breastfeeding $[9,10]$.

Health care suppliers should have adequate knowledge to encourage positive changes in people. Health-related fields "students should be the future role models and breastfeeding supporters, as they gain nutrition information, including infants" ways of feeding, students can also influence social and cultural values and attitudes if they are given the appropriate knowledge and attitudes toward breastfeeding.

Although numerous studies regarding the mothers' knowledge about breastfeeding, a small body of literature addresses the breastfeeding knowledge and attitude among nursing students in Saudi Arabia and other countries in the Gulf region. Despite the many advantages of breastfeeding, breastfeeding rates are below expected rates worldwide and many women continue to use infant formula to feed their babies $[11,12]$.

The study aim was to assess nursing college students' knowledge and attitudes about breastfeeding before and after studying an obstetrics course at a university in Riyadh, Kingdom of Saudi Arabia.

\section{Materials and Methods}

A descriptive, qualitative study was done in March 2017, in the College of Nursing in Riyadh, Kingdom of Saudi Arabia. A convenient sample of students from different levels (94 and 63 students from levels 5 and 7, respectively) was included in the study.

Nursing students from other levels apart from levels 5 and 7 were excluded.

A self-administered questionnaire that was originally developed by Sullivan [13] with some modifications to suit the aim and objectives of the study and to be culturally appropriate was used to assess the students' knowledge and attitudes, and to compare the results of those who studied the obstetric course and those who have not.

The questionnaire consists of five parts; the first part of the questionnaire regards student's knowledge about initiation, duration, and supplementation of breastfeeding. The second part of the questionnaire consists of questions on student's knowledge about the advantages of breastfeeding and formula feeding attitudes. The third part of the questionnaire is about student's knowledge about breastfeeding in special situations e.g. twins, infectious disease of a mother, etc. The fourth part of the questionnaire is about 
the source of student's knowledge about breastfeeding, and the last component of the questionnaire is the demographic data (age, residence, grade, and marital status).

The collected data were analyzed using SPSS. The chi-square test of significance was used for groups' comparison.

\subsection{Ethical Consideration}

The study was reviewed by the university Institutional Review Board (IRB). Informed consent has been taken, and the participants received information about the purpose of the study, content, duration, and significance of the study. The participants' confidentiality was protected.

\section{Results}

The internal consistency of responses was confirmed by Cronbach's alpha (0.9) across questionnaire items.

As shown in Table 1, statistically significant difference between the two levels (level 5 and level 7) with respect to the total score of knowledge.

Table 2 shows that $36.5 \%$ of level 5 students and $47.9 \%$ of level 7 students had good knowledge. Table 3 shows that no statistically significant difference was found in the comparison between the students of level 5 and level 7 concerning the total score of attitude.

TABLE 1. Comparison between level 5 and level 7 with respect to the total score of knowledge

\begin{tabular}{llll}
\hline Total score of knowledge & Level $\mathbf{5}(\boldsymbol{n}=\mathbf{6 3})$ & Level 7 $(\boldsymbol{n}=\mathbf{9 4})$ & $\boldsymbol{P}$-value \\
\hline Mean \pm SD & $18.079 \pm 4.009$ & $19.043 \pm 2.994$ & 0.107 \\
Range (minimum-maximum) & $9-26$ & $10-25$ & \\
\hline
\end{tabular}

TABLE 2. Level 5 and 7 knowledge assessment

\begin{tabular}{llll}
\hline Knowledge & Level 5 & Level 7 & P-value \\
\hline Good knowledge & $23(36.5 \%)$ & $45(47.9 \%)$ & 0.159 \\
Poor knowledge & $40(63.5 \%)$ & $49(52.1 \%)$ & \\
Total & 63 & 94 & \\
\hline
\end{tabular}

TABLE 3. Comparison between level 5 and level 7 students with respect to the total score of attitude

\begin{tabular}{llll}
\hline Total score of attitude & Level $\mathbf{5}(\boldsymbol{n}=\mathbf{6 3})$ & Level 7 $(\boldsymbol{n}=\mathbf{9 4})$ & $\boldsymbol{P}$-value \\
\hline Mean \pm SD & $20.143 \pm 3.417$ & $20.766 \pm 4.007$ & 0.313 \\
Range (minimum-maximum) & $13-32$ & $13-32$ & \\
\hline
\end{tabular}




\section{Discussion}

Studies suggest that well-educated women have a positive attitude toward breastfeeding and they are more likely to breastfeed for a longer duration [11,12]. We conducted this study to evaluate the current level of knowledge of the nursing students towards breastfeeding before and after studying the obstetrics' course and to assess the nursing students' attitudes toward breastfeeding. Students have a certain level of knowledge about the importance and differences of feeding methods that were shown in their answers. The quality of knowledge on breastfeeding was weakened by multiple misunderstandings that are common.

Among nursing students, only 50\% and 39.7\% from level 7 and level 5 respectively had the knowledge that the mother should keep breastfeeding even if she becomes sick or gets an infection, this is consistent with what was mentioned by Cricco-Lizza; that the fearfulness of transferring the disease from the mother to her child if she breastfeeds has a major contribution in this misunderstanding [14]. There are also $85.1 \%$ of level 7 and $85.7 \%$ of level 5 of the students who know that mothers should start breastfeeding in the first few hours after the baby is born. More than $90 \%$ of university students in India and Hong Kong knew mothers should breastfeed within a few hours after the baby's delivery [15].

The nursing students' attitude regarding breastfeeding is neutral and needs to be promoted. Findings by Kim showed that obstetricians have a neutral attitude towards breastfeeding [16], another study done in Malaysia among university medical students, showed that the students' attitude regarding breastfeeding is positive [17].

More than $60 \%$ of both groups showed a positive attitude by disagreeing with the common breastfeeding misconceptions such as that breastfeeding spoil mothers' image and it is only for low socioeconomic status mothers. In 2009, Tan stated that these are some of the common misconceptions that discourage modern women from breastfeeding [18].

In our study, although level 7 nursing students have already studied the obstetrics course, their knowledge of the breastfeeding importance in comparison with level 5 students showed no significant differences. The attitude, on the other hand, was positive and encouraging.

\section{Conclusion}

The result of this study revealed that there is a pressing need to enhance the realization of the significance of breastfeeding should among the nursing students through comprehensive training in the obstetrics' course to fully understand and learn about the benefits of breastfeeding, and to give them the information that help them in mothers education and breastfeeding problems solving.

\section{Acknowledgment}

The authors thank the Deanship of Scientific research/ Princess Nourah Bint Abdurahman University. Also thankful to Kholoud algobs, Haneen Bajabaa, and Amjad Alotibi. 


\section{References}

1. Scariati PD, Grummer-Strawn LM, Fein SB. A longitudinal analysis of infant morbidity and the extent of breastfeeding in the United States. Pediatrics. 1997; 99(6), e5

2. Bachrach VRG, Schwarz E, Bachrach LR. Breastfeeding and the risk of hospitalization for respiratory disease in infancy: a meta-analysis. Archives of Pediatrics \& Adolescent Medicine. 2003; 157(3), 237-243.

3. Oddy WH. Breastfeeding protects against illness and infection in infants and children: a review of the evidence. Breastfeeding Review. 2001; 9(2), 11.

4. Horwood LJ, Fergusson DM. Breastfeeding and later cognitive and academic outcomes. Pediatrics. 1998; 101(1), e9.

5. Weimer J. The economic benefits of breast feeding: a review and analysis. Food assistance and nutrition research Report no. 13. Washington, DC: Food and Rural Economics Division. Economic Research Service, US Department of Agriculture. 2001, 14-18.

6. Dobson B, Murtaugh MA. Position of the American Dietetic Association: breaking the barriers to breastfeeding. Journal of the American Dietetic Association. 2001; 101(10), 1213-1220.

7. Saied H, Mohamed A, Suliman A, Al Anazi W. Breastfeeding knowledge, attitude and barriers among Saudi women in Riyadh. Saudi Medical Journal. 2013; 3(12), 1-9.

8. Al-Sekait M. A study of the factors influencing breastfeeding patterns in Saudi Arabia. Saudi Medical Journal. 1988; 9(6), 596-601.

9. Bernaix LW. Nurses' attitudes, subjective norms, and behavioral intentions toward support of breastfeeding mothers. Journal of Human Lactation. 2000; 16(3), 201-209.

10. Holaday B, Karipis TA, Spicer M. A survey of pediatric nurses' knowledge about breastfeeding. Journal of Pediatric Nursing. 1999; 14(3), 193-200.

11. Leung T, Tam W, Hung E, Fok T, Wong G. Socio demographic and atopic factors affecting breastfeeding intention in Chinese mothers. Journal of Paediatrics and Child Health. 2003; 39(6), 460-464.

12. Dennis CL. Breastfeeding initiation and duration: a 1990-2000 literature review. Journal of Obstetric, Gynecologic, \& Neonatal Nursing. 2002; 31(1), 12-32.

13. Jepson-Sullivan RM, Metos J, Smith E, Jordan K. Reliability and validity of a breastfeeding knowledge survey in allied health students. Journal of the American Dietetic Association. 2011; 111(9), A68.

14. Cricco-Lizza R. Student nurses' attitudes and beliefs about breast-feeding. Journal of Professional Nursing. 2006; 22(5), 314-321.

15. Kapil U, Manocha S. Knowledge and attitude towards breast feeding among adolescent girls. The Indian Journal of Pediatrics. 1990; 57(3), 401-404.

16. Kim HS. Attitudes and knowledge regarding breast-feeding: a survey of obstetric residents in metropolitan areas of South Korea. Southern Medical Journal. 1996; 89(7), 684-688.

17. Mohamad N, Saddki N, Azman KNK, Aziz IDA. Knowledge, attitude, exposure, and future intentions toward exclusive breastfeeding among Universiti Sains Malaysia final year medical and dental students. Korean Journal of Family Medicine. 2019; 40(4), 261-268.

18. Tan K. Knowledge, attitude and practice on breastfeeding in Klang, Malaysia. International Medical Journal. 2009; 8(1), 17-22. 